\title{
Sinusoidal Electrical Pulse More Efficiently Evokes Retinal Excitation than Rectangular Electrical Pulse in Retinal Prostheses
}

\author{
Yukari Nakano, ${ }^{1 *}$ Yasuo Terasawa,${ }^{1,2}$ Hiroyuki Kanda,${ }^{3}$ Hiroyuki Tashiro, ${ }^{4}$ \\ Koji Osawa, ${ }^{5}$ Tomomitsu Miyoshi, ${ }^{6}$ Hajime Sawai, ${ }^{7}$ and Takashi Fujikado ${ }^{3}$ \\ ${ }^{1}$ Artificial Vision Institute, Research and Development Division, Nidek Co., Ltd., \\ 13-2 Hama-cho, Gamagori, Aichi 443-0036, Japan \\ ${ }^{2}$ Graduate School of Materials Science, Nara Institute of Science and Technology, \\ 8916-5 Takayama-cho, Ikoma, Nara 630-0192, Japan \\ ${ }^{3}$ Department of Applied Visual Science, Osaka University Graduate School of Medicine, \\ 2-2 Yamadaoka, Suita, Osaka 565-0871, Japan \\ ${ }^{4}$ Department of Health Sciences, Faculty of Medical Sciences, Kyushu University, \\ 3-1-1 Maidashi, Higashi-ku, Fukuoka 812-8582, Japan \\ ${ }^{5}$ Artificial Retina Development Office, Development Division, Nidek Co., Ltd., \\ 34-14 Maehama, Hiroishi-cho, Gamagori, Aichi 443-0038, Japan \\ ${ }^{6}$ Department of Integrative Physiology, Osaka University Graduate School of Medicine, \\ 2-2 Yamadaoka, Suita, Osaka 565-0871, Japan \\ ${ }^{7}$ Department of Health Sciences, School of Nursing, Osaka Prefecture University, \\ 3-7-30 Habikino, Habikino, Osaka 583-8555, Japan
}

(Received March 31, 2017; accepted June 23, 2017)

Keywords: retinal prosthesis, stimulation waveform, electrical stimulation parameter, retinitis pigmentosa, threshold charge

Retinal prostheses for blindness due to retinal photoreceptor degeneration stimulate the retina electrically to evoke a pseudolight sensation (phosphenes). Although rectangular pulses of electrical stimulation are commonly used in retinal prostheses, it remains unclear whether nonrectangular pulses are effective in evoking this response. Here, we conducted in vivo electrophysiological experiments to compare the effectiveness of sinusoidal and rectangular pulses. Biphasic sinusoidal pulses (cathodic-first) applied suprachoroidally and transretinally to the rat eye elicited larger field responses in the superior colliculus than did rectangular pulses. The threshold charge for the evoked response of the sinusoidal pulse was significantly lower than that of the rectangular pulse, suggesting that a sinusoidal pulse is more effective than a rectangular pulse in our retinal prosthesis. Because a sinusoidal pulse can evoke phosphenes with a small charge magnitude even if the electrode area is reduced, the charge density does not increase; thus, the pulse can stimulate the retina without causing tissue injury. Because the sinusoidal pulse allows us to reduce the electrode area, it is possible that phosphenes can be localized to a smaller area by limiting the range of stimulated retinal ganglion cells. Therefore, high resolution of retinal prostheses using the sinusoidal pulse can be expected.

"Corresponding author: e-mail: yukari_nakano@nidek.co.jp http://dx.doi.org/10.18494/SAM.2017.1611 


\section{Introduction}

The recent development of brain-machine interfaces (BMIs) is promising for the treatment of numerous disabilities. Such technology may extend patients' abilities and overcome handicaps in their daily living activities via devices that directly connect to the nervous system. BMIs are classified into two major categories: output BMI and input BMI. ${ }^{(1)}$ Output BMIs decode neural outputs from the brain to control devices such as robotic arms. ${ }^{(2)}$ Sensor devices receive external signals such as sound and light and transmit them to the sensory nervous system. Input BMIs are used for sensory disabilities such as blindness and deafness. The sensor devices receive external signals and provide the sensory nervous system with corresponding inputs. The most successful example of an input BMI is the cochlear implant for neurosensory deafness. An electrode array implanted into the degenerated cochlea electrically stimulates the cochlear nerve fibers to provide auditory sensation. This device has been in practical use for more than 30 years and has reconstructed the hearing of hundreds of thousands of people worldwide. ${ }^{(3)}$ The visual equivalent of the cochlear implant is the retinal prosthesis. The target disease of the retinal prosthesis is retinitis pigmentosa (RP), a degenerative disease that is one cause of acquired blindness and affects 1 in 4000 people worldwide. ${ }^{(4)}$ This incurable disease involves the progressive degeneration of retinal photoreceptor cells. At present, no fundamental therapy for RP has been established. Retinal prostheses have been studied as a means of restoring the visual function lost to RP. One of the retinal prostheses has been approved recently in the United States and Europe. ${ }^{(5)}$

The retinal prosthesis converts an image captured by a camera into electrical signals. These signals stimulate the electrode array in the retinal implant to emit electrical signals to the retina. This retinal stimulation evokes a pseudolight sensation called phosphenes, finally restoring vision artificially. While RP results in severe damage to photoreceptor cells, other types of inner retinal cells, such as retinal ganglion cells, remain even after blindness ensues. ${ }^{(6)}$ This observation suggests that vision could be reconstructed through electrical stimulation of the remaining retinal cells. Retinal prostheses are based on this principle. Retinal prostheses are classified into four types, depending on the position of the electrode array: (1) epiretinal (placed on the retina from the vitreous side), ${ }^{(5)}(2)$ subretinal (placed between the choroid and the retina), ${ }^{(7)}$ (3) placed between the choroid and retinal pigment epithelium, ${ }^{(8)}$ and (4) placed in the sclera. ${ }^{(9)}$ We have been developing a retinal prosthesis based on suprachoroidal transretinal stimulation (STS) in which the electrode array is placed in a scleral pocket created by making a half-layer incision. ${ }^{(9)}$ By this method, the fixation of the electrode array is more mechanically stable than that of other methods. Further advantages are that the surgical invasion of the eye is small and a wide field of view can be secured. However, since retinal cells are stimulated via the choroid, the stimulation charge required to induce light sensation is greater than that of other approaches. ${ }^{(10)}$ This problem might be solved by investigating the stimulation parameters.

A biphasic rectangular current pulse is generally used for electrical stimulation in retinal prostheses, regardless of the implantation position of the stimulating electrode array. For example, biphasic rectangular current pulses are used in epiretinal, ${ }^{(5)}$ suprachoroidal, ${ }^{(8)}$ and STS prostheses..${ }^{(9,11)}$ Charge-balanced biphasic pulses can carry out electrical stimulation safely without causing damage to tissues. ${ }^{(12)}$ The optimum pulse duration and frequency for retinal ganglion cell activation (with respect to stimulation effectiveness) has been studied in vitro. ${ }^{(13-15)}$ However, the optimal stimulation waveform shape remains unknown. Accordingly, a rectangular pulse 
may not be optimal as the stimulation waveform. Stimulation waveforms that reduce the required threshold charge could allow for the realization of smaller electrodes with higher spatial resolution. Therefore, determining the optimal waveform for high stimulation effectiveness is important for the development of retinal prostheses. In a study of rat retina in vitro, Weitz et al. reported that a sinusoidal pulse has a lower threshold charge than does a rectangular one. ${ }^{(13)}$ However, this result was obtained using epiretinal stimulation; therefore, the effect of a sinusoidal pulse in STS has not been established. In addition, these in vitro results may not hold true in vivo. Therefore, we conducted in vivo electrophysiological experiments to determine whether a sinusoidal pulse is also effective in STS and in vivo.

\section{Materials and Methods}

\subsection{Animals}

We used normal rats (Long-Evans; $n=13$ ) aged 15-24 weeks and retinal-degenerated rats [Royal College of Surgeons (RCS); $n=20$ ] aged 30-37 weeks. All procedures were in accordance with the Association for Research in Vision and Ophthalmology (ARVO) Statement for the Use of Animals in Ophthalmic and Vision Research. This study was approved by the Institutional Animal Care Committee of Nidek.

\subsection{Surgical preparation}

All animals were anesthetized for surgery and acute electrophysiological recordings by intraperitoneal injection of urethane $(1.75 \mathrm{~g} / \mathrm{kg})$. The head of the rat was fixed with a stereotaxic apparatus (SR-5R-HT, Narishige Science Institute Laboratory, Tokyo, Japan). The left eye of the rat was exposed, and the partial sclera of the eye was resected surgically from approximately $1 \mathrm{~mm}$ below the optic nerve to the vicinity of the equator. A platinum stimulating electrode (diameter, 0.5 $\mathrm{mm}$; height, $0.3 \mathrm{~mm}$ ) was placed in the partial scleral resection using a micromanipulator (SF-1, S, Tokyo, Japan). A return electrode (platinum-iridium wire; diameter, $0.2 \mathrm{~mm}$ ) was placed into the vitreous body using a micromanipulator (Minimanisuke, S). After partial removal of the sclera, mineral oil was used around the eye to prevent drying. A craniotomy was performed to remove the posterior two-thirds of the right parietal bone, and the underlying cortex was gently aspirated to expose the dorsal surface of the right superior colliculus (SC). Mineral oil was applied to the exposed SC area during the experiment to prevent drying. A stainless-steel screw acting as the reference electrode was inserted into the occipital bone.

\subsection{Photic stimulation}

To evaluate the survival of photoreceptors in the retina of RCS rats, we recorded visual evoked potentials from the SC. The left eye of the rat was dilated with a mydriatic agent (Mydrin-P ophthalmic solution, Santen Pharmaceutical, Osaka, Japan), and the cornea surface was covered with a contact lens electrode combined with a light-emitting diode (LED) light source (R250 LEDT, Mayo, Aichi, Japan). After dark adaptation for $20 \mathrm{~min}$, flashing light stimuli (luminance, $1000 \mathrm{~cd} / \mathrm{m}^{2}$; duration, $1 \mathrm{~ms}$; frequency, $1 \mathrm{~Hz}$ ) were applied in a dark room using an LED (LS-100, Mayo). 


\subsection{Electrical stimulation}

Figure 1 shows the setup for electrical stimulation. A single biphasic current pulse was applied between the stimulating electrode and the return electrode using a stimulus generator (STG 4002, Multi Channel Systems, Reutlingen, Germany). The current pulse duration was $0.5 \mathrm{~ms}$, and the stimulation frequency was $1 \mathrm{~Hz}$. Electrical stimulation was performed using rectangular and sinusoidal pulses of the single biphasic current pulse (outward current/cathodic-first) (Fig. 2). The current amplitudes ranged from $50 \mu \mathrm{A}$ to $1 \mathrm{~mA}$. The stimulation waveforms were confirmed using an oscilloscope (TPS 2014, Tektronix, Oregon, USA).

\subsection{Electrophysiological recordings}

A tungsten microelectrode [resistance, $0.5 \mathrm{M} \Omega$; diameter, approximately $5.0 \mu \mathrm{m}$ (uninsulated part), Unique Medical, Tokyo, Japan] was inserted to the depth of $250 \mu \mathrm{m}$ from the SC surface using a micromanipulator (MMO-203, Narishige) to record local field potentials evoked by photic and electrical stimulation. The recorded potentials were amplified using a differential amplifier (DP-301, Warner, Connecticut, USA) (amplification: photic stimulation, 100 times; electrical stimulation, 1000 times; band-pass filter, $10 \mathrm{~Hz}-3 \mathrm{kHz}$ ) and then digitally sampled and averaged using PowerLab (2/26, ML 826, ADInstruments, Dunedin, New Zealand) with evoked potentials (EPs) collection software (LabChart, ADInstruments). The signal for photic stimulation was averaged 50 times for normal rats and 300 times for RCS rats. The signal averaging for electrical stimulation

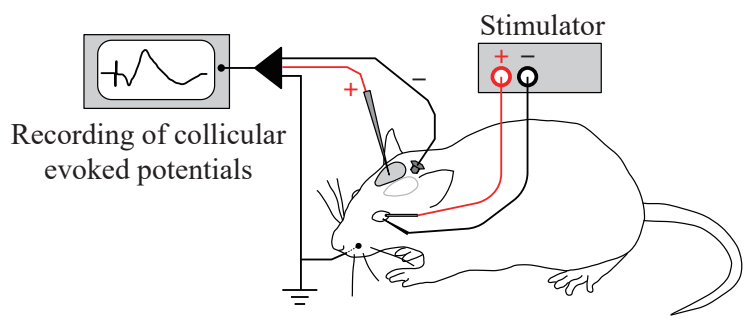

Fig. 1. (Color online) Setup for electrical stimulation and recording. Under anesthesia, a platinum stimulating electrode (diameter, $0.5 \mathrm{~mm}$; height, $0.3 \mathrm{~mm}$ ) was set into a partial scleral resection of the rat eye. A return electrode (platinum-iridium wire; diameter, $0.2 \mathrm{~mm}$ ) was placed into the vitreous body. The single biphasic current pulse (outward current/cathodic-first) was applied between the stimulating electrode and the return electrode. The potential evoked by electrical stimulation was recorded with a needle electrode [tungsten; resistance, $0.5 \mathrm{M} \Omega$; diameter, approximately $5.0 \mu \mathrm{m}$ (uninsulated part)] in the superior colliculus.

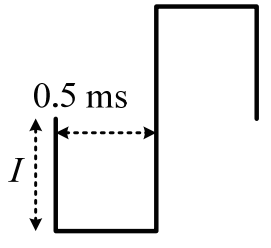

(a)

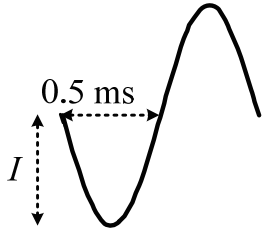

(b)

Fig. 2. Waveforms of electrical stimulation: (a) rectangular pulse and (b) sinusoidal pulse. Both are biphasic symmetric pulses (outward current first; i.e., cathode-first). The current pulses of the two types of waveforms had a duration of $0.5 \mathrm{~ms}$ and current amplitude ranging from $50 \mu \mathrm{A}$ to $1 \mathrm{~mA}$. 
was 50 times for both normal and RCS rats. The position of the largest EP was determined by moving the recording electrode along the rostral-caudal or medio-lateral axis of the SC at an interval of 100-250 $\mu \mathrm{m}$. The position with the maximum EP for electrical stimulation was set as the point corresponding to the retinal location that was electrically stimulated. Subsequent recordings were completed at this position.

\subsection{Data analysis}

The average waveform after 50 signal averages of the EP was evaluated. For the analysis, we used the difference (P1-N1) between the first positive wave (P1) and the subsequent first negative wave (N1) at approximately 5 and $10 \mathrm{~ms}$ after stimulation. EP amplitudes (P1-N1) were plotted against the charge magnitude [Fig. 3(a)]. The regression line was calculated in the range between $E_{1}$ [Eq. (1)] and $E_{2}$ [Eq. (2)] [Fig. 3(a)]. $E_{1}$ and $E_{2}$ are the ranges in which the averaged coefficient of determination of the regression line is maximal in all individuals. MAX was defined as the largest EP [Fig. 3(a)]. The threshold charge was calculated as the intersection of the $X$-axis and the regression line [Fig. 3(b)].

$$
\begin{gathered}
E_{1}=M A X \times 0.6 \\
E_{2}=M A X \times 0.05
\end{gathered}
$$

A paired $t$-test was used to assess the differences in the threshold charge between stimulus waveform shapes. The significance level was set at $p<0.05$. Of the recorded data, we used amplitudes for which the coefficient of determination of the regression lines was greater than 0.9 for statistical analysis. $\mathrm{R}$ version 3.3.2 $2^{(16)}$ was used for the statistical analysis.

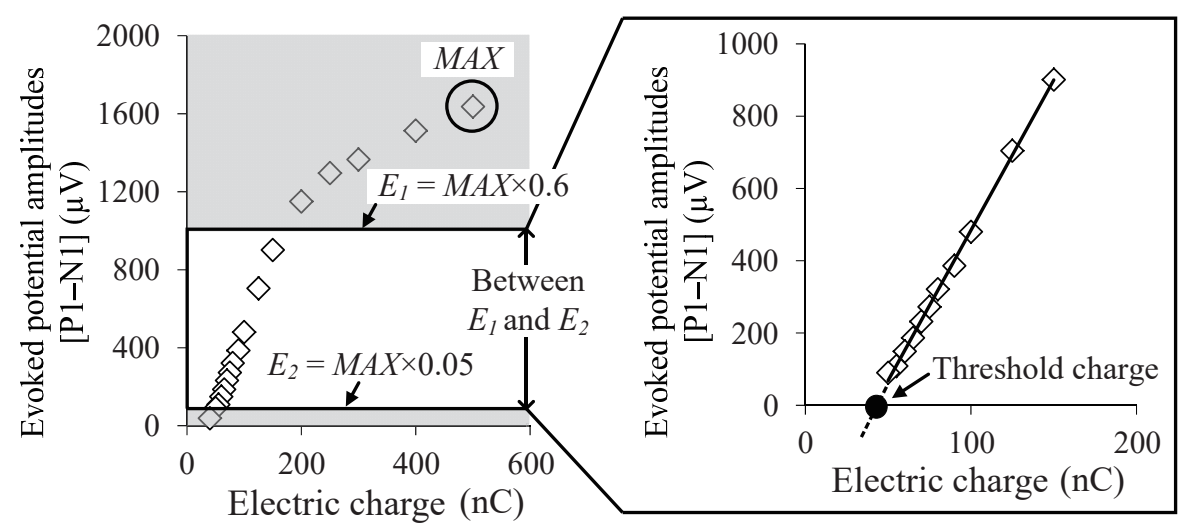

(a)

(b)

Fig. 3. Calculation of threshold charge. (a) EP amplitudes were plotted against the electric charge magnitude. (b) Magnified view of the region between $E_{1}$ and $E_{2}$. The regression line was calculated in the range between $E_{1}$ and $E_{2}$. The threshold charge was calculated as the intersection of the $X$-axis and the regression line. The ranges of $E_{1}$ and $E_{2}$ were set as those where linearity was obtained for all individuals. MAX was defined as the largest EP. 


\subsection{Histological analysis}

After completion of the experiment, rats were euthanized with an overdose of sodium pentobarbital. The eyes were enucleated immediately after euthanasia in four rats. Two normal rats and two RCS rats were randomly selected for histological analysis. The extracted eyes were fixed for $24 \mathrm{~h}$ with a solution of $1.5 \%$ glutaraldehyde and $3 \%$ formaldehyde in phosphate buffer. Next, the eyes were fixed with a neutral buffered formalin solution for 5-7 d. After embedding in resin, the eyes were prepared into slices with thicknesses of 4-5 $\mu \mathrm{m}$. The sliced tissues were stained with hematoxylin and eosin (HE). The layered structure of the outer retina of normal and RCS rats was histologically observed under a microscope.

\section{Results}

\subsection{Waveform of electrical stimulation}

The actual waveform of the rectangular pulse confirmed with the oscilloscope is shown in Fig. 4. In the case of a rectangular wave, overshoot or undershoot, a phenomenon in which the waveform exceeds the base line and becomes a steady value, may occur at the rising and falling portions of the waveform, respectively. Under the stimulation condition of this study, neither overshoot nor undershoot was observed.

\subsection{Response waveform}

While EPs were clearly obtained in all normal rats 30 to $100 \mathrm{~ms}$ after photic stimulation [Fig. 5(a)], EPs after photic stimulation were not observed in any of the RCS rats [Fig. 5(b)]. This result is likely due to the complete degeneration of photoreceptor cells typically seen in RCS rats. As we previously reported ${ }^{(17)}$ and also observed in this study, a rectangular pulse of electrical current $(>100 \mu \mathrm{A})$ applied to the eye globe elicited EPs composed of an initial positive wave (P1) and a subsequent first negative wave (N1) in both normal and RCS rats (representative example, Fig. 6). Peak latencies of $\mathrm{P} 1$ and $\mathrm{N} 1$ are approximately 5 and $10 \mathrm{~ms}$ after stimulation, respectively.

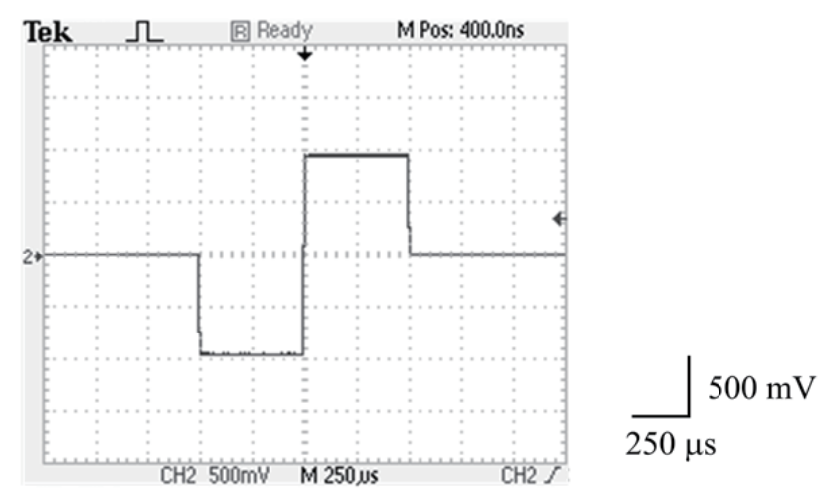

Fig. 4. Actual waveform of the rectangular pulse simulated. It is 16 times the average waveform when stimulated with $1 \mathrm{~mA}$. Under the stimulation condition of this study, neither overshoot nor undershoot was observed. 


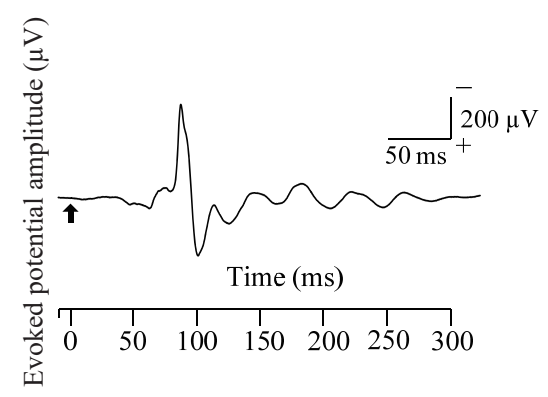

(a)

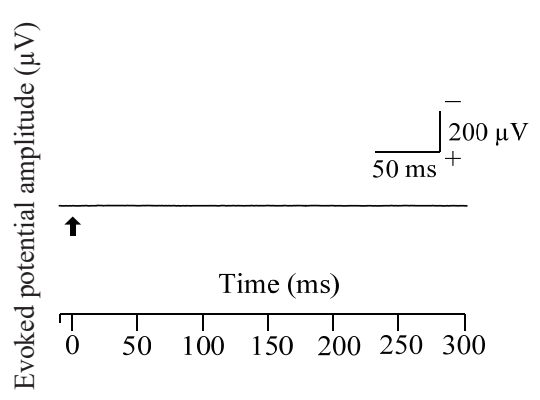

(b)

Fig. 5. Example of response waveforms observed by photic stimulation in (a) normal rats (Long-Evans) and (b) RCS rats. The artifacts of photic stimulation, indicated by arrows, show the application of the light flash. The artifact appearing upon irradiation with the light flash was as small as $20 \mu \mathrm{V}$ and could not be clearly confirmed. Peaks of EP were observed about 30-100 ms after stimulation in all normal rats. In contrast, no response to photic stimulation was observed in any of the RCS rats. This observation indicates that the photoreceptor cells in RCS rats were degraded.

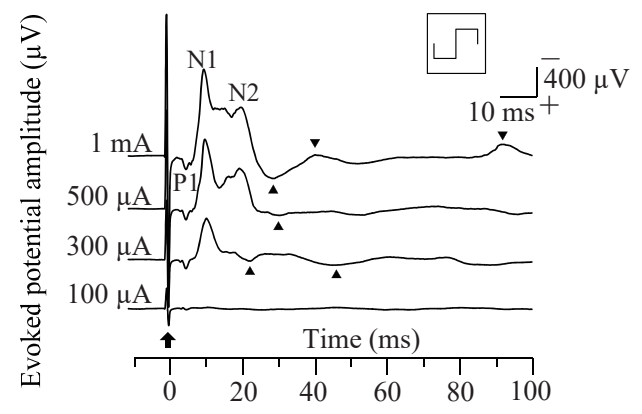

(a)

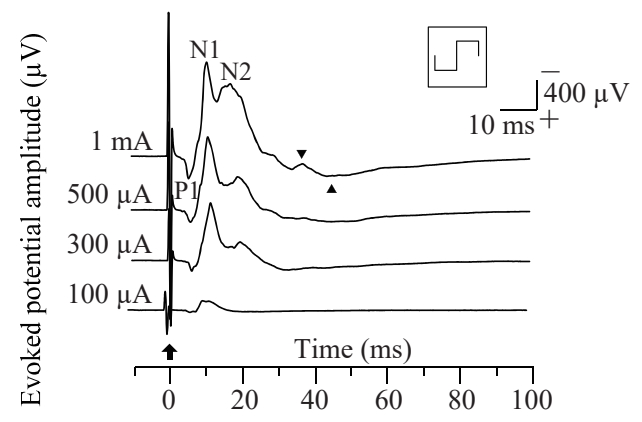

(c)

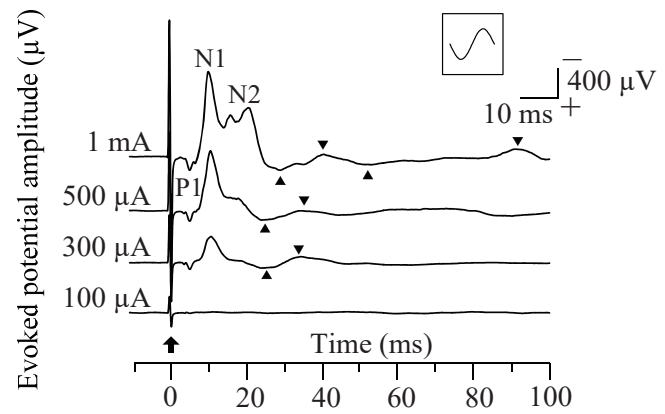

(b)

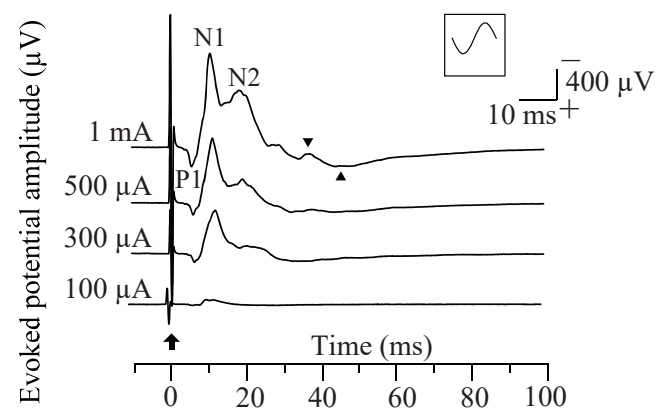

(d)

Fig. 6. Example of the response waveforms observed after electrical stimulation of $1 \mathrm{~mA}, 500 \mu \mathrm{A}, 300 \mu \mathrm{A}$, and $100 \mu \mathrm{A}$. (a) Normal rat, rectangular pulse, (b) normal rat, sinusoidal pulse, (c) RCS rat, rectangular pulse, and (d) RCS rat, sinusoidal pulse. The artifacts of electrical stimulation, indicated by arrows, show the application of the current pulse. Regardless of the stimulation waveform shape at a current amplitude of $100 \mu \mathrm{A}$ or more, the EPs were composed of the first positive wave and the subsequent negative wave at approximately 5.0 and $10 \mathrm{~ms}$ after stimulation, respectively, in all normal rats. For $1 \mathrm{~mA}$ electrical stimulation, regardless of the stimulation waveform shape, the second negative peak, N2, was observed at approximately 10 to $25 \mathrm{~ms}$ in all rats. N2 disappeared as the stimulation current amplitude decreased. Gentle peaks observed after $20 \mathrm{~ms}$ are indicated by triangles. The gentle peaks disappeared as the stimulation current amplitude decreased. 
As shown in Figs. 6(b) and 6(d), the sinusoidal pulse was also able to generate P1-N1 responses in normal as well as RCS rats. Accordingly, we assumed that the response waveform obtained in this study was not an artifact or noise but rather a response to the electrical stimulation. For $1 \mathrm{~mA}$ electrical stimulation, regardless of the stimulation waveform shape, the second negative peak (N2) was observed at approximately $10-25 \mathrm{~ms}$ in all normal and RCS rats (Fig. 6). As the stimulation current amplitude decreased, N2 disappeared in both the normal and RCS rats, regardless of the stimulation waveform. For all normal rats, a stimulation of $100 \mu \mathrm{A}$ or more resulted in a gentle peak at approximately 20-90 ms, regardless of the stimulation waveform shape [Figs. 6(a) and 6(b)]. However, except for stimulation with $1 \mathrm{~mA}$, peaks at $20 \mathrm{~ms}$ after stimulation were not observed in any of the RCS rats [Figs. 6(c) and 6(d)].

\subsection{Threshold charge}

The EP amplitudes (P1-N1) are shown in Fig. 7. Of the 20 RCS rats tested, 7 had a coefficient of determination of a partial regression line less than 0.9 and were excluded from the data analysis. In both normal and RCS rats, the threshold charge in the sinusoidal pulse was significantly lower than that of the rectangular pulse (normal rats: rectangular, $39.6 \pm 10.2 \mathrm{nC}$; sinusoidal, $32.9 \pm 8.7 \mathrm{nC} ; p<0.01$; RCS rats: rectangular, $27.0 \pm 16.7 \mathrm{nC}$; sinusoidal, $24.1 \pm 13.8 \mathrm{nC} ; p<0.05$ ) [mean \pm standard deviation (SD), paired $t$-test]. The mean threshold charge of the sinusoidal pulse was $17 \%$ lower than that of the rectangular pulse in normal rats. In RCS rats, the threshold charge was $11 \%$ lower with the sinusoidal pulse than with the rectangular pulse.

\subsection{Histological analysis}

An example of tissue specimens from normal rats and RCS rats is shown in Fig. 8. Analysis of tissue specimens of RCS rats revealed that the layered structures of the outer retina were completely deteriorated [Fig. 8(b)]. This observation suggested that the photoreceptor cells of RCS rats were sufficiently degenerated. In the two RCS rats for which the tissues were observed, the

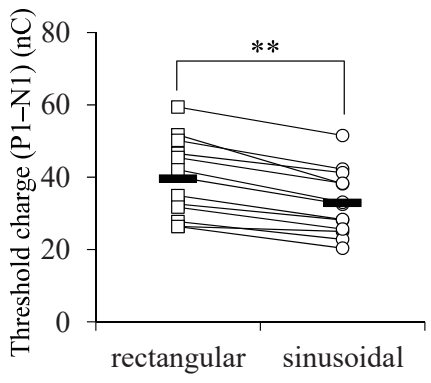

(a)

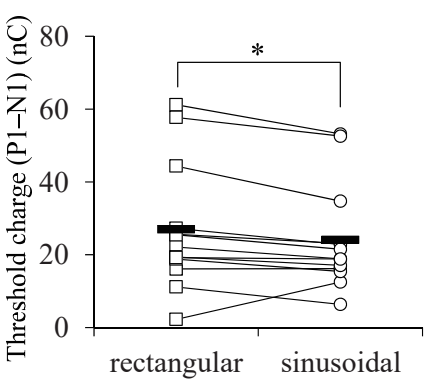

(b)

Fig. 7. Threshold charges in (a) normal rats (Long-Evans) $(n=13)$ and (b) RCS rats $(n=13)$. The black horizontal bars (-) indicate the mean threshold charge. The threshold values for each animal are indicated by squares $(\square)$ for rectangular stimulation and circles ( $($ ) for sinusoidal stimulation. Lines connecting the symbols indicate that the data are from the same individual. Paired $t$-test $\left({ }^{*} p<0.05 ;{ }^{*} p<0.01\right)$ was used to detect significant differences. The threshold charge was significantly lower for the sinusoidal pulse than for the rectangular pulse $(p<0.05)$. 


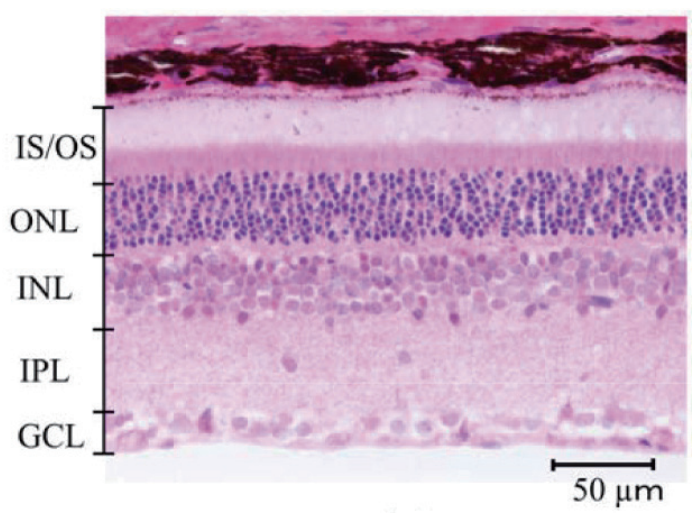

(a)

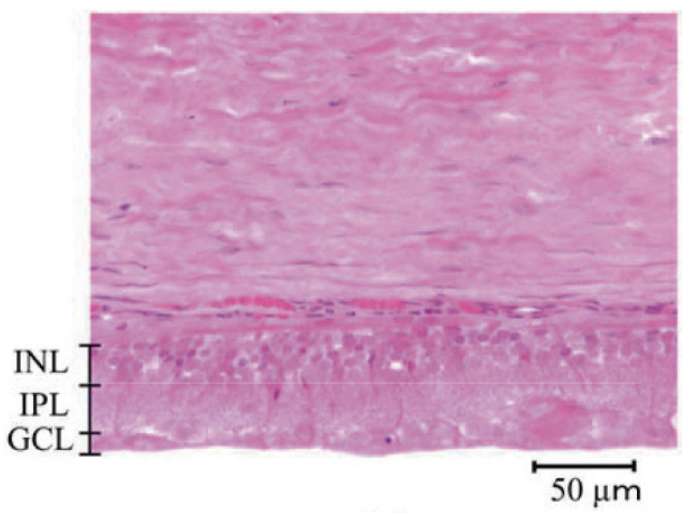

(b)

Fig. 8 (Color online) Representative tissue specimens of (a) normal 23-week-old rats (Long-Evans) and (b) 34-week-old RCS rats. The black bars represent $50 \mu \mathrm{m}$. The inner segment/outer segment (IS/OS), outer nuclear layer (ONL), INL, inner plexiform layer (IPL), and GCL are indicated. Panel (b) confirms that the outer retina i absent in RCS rats.

layered structures of the outer retina could not be detected. However, the layered structure in the inner retina [from the inner nuclear layer (INL) to the ganglion cell layer (GCL)] was observed [Fig. 8(b)]. Both the inner and outer retinal layers were observed in the histological analysis of the two normal rats [Fig. 8(a)].

\section{Discussion}

We evaluated the differences between the threshold charges of rectangular and sinusoidal pulses. Under the stimulation conditions of this study, with rectangular pulse stimulation, overshoot and undershoot were not observed. Therefore, it is considered that the rectangular wave used in this study was stimulated with a waveform close to the ideal shape (Fig. 4). The EP amplitude used for calculating the threshold charge was defined as the difference between P1 and $\mathrm{N} 1$, the peaks obtained upon stimulation with a current greater than $100 \mu \mathrm{A}$, regardless of the stimulation waveform shape. Therefore, we focused on P1 and N1.

In both normal and RCS rats, the mean threshold charge of the sinusoidal pulse was significantly lower than that of the rectangular pulse. Weitz et al. reported that the threshold charge at which retinal ganglion cells fired was $22.8 \pm 35.5 \%$ greater for a rectangular pulse than for a sinusoidal pulse. ${ }^{(13)}$ In their in vitro study, they applied direct stimulation to the ganglion cell side of the retina. Although this methodology differed from ours, the results are similar. From this observation, we conclude that the sinusoidal pulse is more effective than the rectangular pulse, regardless of the implantation site of the stimulating electrode. Because a sinusoidal pulse with a small charge magnitude can evoke phosphenes, even if the electrode area is reduced, the charge density does not increase and can stimulate the retina without causing tissue injury. Sekirnjak et al. reported that reducing the electrode area can limit the range of stimulated retinal ganglion cells to a spatially confined region. ${ }^{(14)}$ The localization of stimulation indicates that the excitation of the retina and the primary visual cortex are both localized. Thus, the localization of phosphenes is suggested to be achieved by optimizing the stimulation waveform. 
Efforts to improve the resolution of retinal prostheses using finer electrodes results in a decrease in the charge injection capability (CIC). The CIC is the maximum injectable charge that does not cause an irreversible electrochemical reaction. ${ }^{(18)}$ The use of smaller electrodes raises the concern that the CIC will be lower than that of a large electrode. By lowering the threshold charge through the use of a sinusoidal waveform, electrical stimulation can be safely applied, even if using smaller electrodes. The CIC for rectangular pulse stimulation is reported by Terasawa et al., ${ }^{(18)}$ but that of sinusoidal pulse stimulation is unknown. Confirmation that sinusoidal pulses do not cause an irreversible electrochemical reaction on the electrode surface will be needed in the future. Conveniently, a sinusoidal pulse can be easily produced using electronic circuits.

This study is the first to show the effectiveness of a sinusoidal pulse in vivo using STS. Results are promising for future clinical applications of this method. Both functional evaluations based on photic stimulation and on histological analyses of tissue samples indicate photoreceptor degeneration in all RCS rats [Figs. 5(b) and 8(b)]. The effectiveness of the sinusoidal pulse in RCS rats suggests that this method would be efficacious for patients suffering from RP. To facilitate the clinical use of the sinusoidal pulse, we will conduct further assessments of safety, including the evaluation of electrochemical reactions on the electrode surface upon stimulation with threshold charge sinusoidal pulses, and investigate the effect of chronic stimulation with sinusoidal pulses and their influence on neural tissue.

\section{Conclusions}

The threshold charge of the sinusoidal pulse is smaller than that of the rectangular pulse. Because a sinusoidal pulse can evoke phosphenes with a small charge magnitude even if the electrode area is reduced, the charge density does not increase; thus, the pulse can stimulate the retina without causing tissue injury. The sinusoidal pulse allows us to reduce the electrode area, and it is possible that phosphenes can be localized to a smaller area by limiting the range of stimulated retinal ganglion cells. Therefore, high resolution of retinal prostheses using the sinusoidal pulse can be expected. Our results suggest that the sinusoidal pulse is effective for use in retinal prosthetic devices.

\section{References}

1 H. Yokoi, K. Sato, S. Morishita, T. Nakamura, R. Kato, T. Umeda, H. Watanabe, Y. Nishimura, T. Isa, K. Ikoma, T. Miyamoto, and O. Yamamura: Advances in Therapeutic Engineering, ed. W. Yu (CRC Press, Boca Raton, 2012) p. 219.

2 M. Hirata, T. Yanagisawa, K. Matsushita, H. Sugata, Y. Kamitani, T. Suzuki, H. Yokoi, T. Goto, M. Shayne, Y. Saitoh, H. Kishima, M. Kawato, and T. Yoshimine: Technological Advancements in Biomedicine for Healthcare Applications, ed. J. Wu (Medical Information Science Reference, Hershey, 2012) p. 362.

3 NIDCD Information Clearinghouse: NIDCD Fact Sheet Cochlear Implants, NIH Publication No. 11-4798 (2016).

4 D. T. Hartong, E. L. Berson, and T. P. Dryja: The Lancet 368 (2006) 1795.

5 D. H. Ghodasra, A. Chen, J. F. Arevalo, D. G. Birch, K. Branham, B. Coley, G. Dagnelie, E. de Juan, R. G. Devenyi, J. D. Dorn, A. Fisher, D. R. Geruschat, N. Z. Gregori, R. J. Greenberg, P. Hahn, A. C. Ho, A. Howson, S. S. Huang, R. Iezzi, N. Khan, B. L. Lam, J. I. Lim, K. G. Locke, M. Markowitz, A.-M. Ripley, M. Rankin, H. Schimitzek, F. Tripp, J. D. Weiland, J. Yan, D. N. Zacks, and K. T. Jayasundera: BMC Ophthalmol. 16 (2016) 1.

6 A. Santos, M. S. Humayun, E. de Juan Jr., R. J. Greenburg, M. J. Marsh, I. B. Klock, and A. H. Milam: Arch. Ophthalmol. 115 (1997) 511. 
7 K. Stingl, K. U. Bartz-Schmidt, D. Besch, C. K. Chee, C. L. Cottriall, F. Gekeler, M. Groppe, T. L. Jackson, R. E. MacLaren, A. Koitschev, A. Kusnyerik, J. Neffendorf, J. Nemeth, M. A. Naeem, T. Peters, J. D. Ramsden, H. Sachs, A. Simpson, M. S. Singh, B. Wilhelm, D. Wong, and E. Zrenner: Vision Res. 111 (2015) 149.

8 N. C. Sinclair, M. N. Shivdasani, T. Perera, L. N. Gillespie, H. J. McDermott, L. N. Ayton, and P. J. Blamey: Invest. Ophthalmol. Vis. Sci. 57 (2016) 4948.

9 T. Fujikado, M. Kamei, H. Sakaguchi, H. Kanda, T. Endo, M. Hirota, T. Morimoto, K. Nishida, H. Kishima, Y. Terasawa, K. Oosawa, M. Ozawa, and K. Nishida: Invest. Ophthalmol. Vis. Sci. 57 (2016) 6147.

10 T. Morimoto, M. Kamei, K. Nishida, H. Sakaguchi, H. Kanda, Y. Ikuno, H. Kishima, T. Maruo, K. Konoma, M. Ozawa, K. Nishida, and T. Fujikado: Invest. Ophthalmol. Vis. Sci. 52 (2011) 6785.

11 Y. Nakano, Y. Terasawa, H. Tashiro, K. Osawa, M. Ozawa, and T. Fujikado: Invest. Ophthalmol. Vis. Sci. $56(2015) 778$.

12 S. F. Cogan: Annu. Rev. Biomed. Eng. 10 (2008) 275.

13 A. C. Weitz, D. Nanduri, M. R. Behrend, A. Gonzalez-Calle, R. J. Greenberg, M. S. Humayun, R. H. Chow, and J. D. Weiland: Sci. Transl. Med. 7 (2015) 203.

14 C. Sekirnjak, P. Hottowy, A. Sher, W. Dabrowski, A. M. Litke, and E. J. Chichilnisky: J. Neurophysiol. 95 (2006) 3311.

15 P. Twyford and S. Fried: IEEE Trans. Neural Syst. Rehabil. Eng. 24 (2016) 413.

16 R Core Team: The R Project for Statistical Computing, https://www.r-project.org/ (accessed November 2016).

17 H. Kanda, T. Morimoto, T. Fujikado, Y. Tano, Y. Fukuda, and H. Sawai: Invest. Ophthalmol. Vis. Sci. 45 (2004) 560.

18 Y. Terasawa, H. Tashiro, Y. Nakano, K. Osawa, and M. Ozawa: Conf. Proc. IEEE Eng. Med. Biol. Soc. (2013) p. 3567. 